\title{
Validation List no. 125 \\ List of new names and new combinations previously effectively, but not validly, published
}

\author{
Correspondence \\ Jean Euzéby \\ email address can be found at \\ www.bacterio.net
}

\begin{abstract}
The purpose of this announcement is to effect the valid publication of the following effectively published new names and new combinations under the procedure described in the Bacteriological Code (1990 Revision). Authors and other individuals wishing to have new names and/or combinations included in future lists should send three copies of the pertinent reprint or photocopies thereof, or an electronic copy of the published paper, to the IJSEM Editorial Office for confirmation that all of the other requirements for valid publication have been met. It is also a requirement of IJSEM and the ICSP that authors of new species, new subspecies and new combinations provide evidence that types are deposited in two recognized culture collections in two different countries (i.e. documents certifying deposition and availability of type strains). It should be noted that the date of valid publication of these new names and combinations is the date of publication of this list, not the date of the original publication of the names and combinations. The authors of the new names and combinations are as given below, and these authors' names will be included in the author index of the present issue. Inclusion of a name on these lists validates the publication of the name and thereby makes it available in bacteriological nomenclature. The inclusion of a name on this list is not to be construed as taxonomic acceptance of the taxon to which the name is applied. Indeed, some of these names may, in time, be shown to be synonyms, or the organisms may be transferred to another genus, thus necessitating the creation of a new combination.
\end{abstract}

\begin{tabular}{|c|c|c|c|c|}
\hline Name/author(s) & Proposed as: & Nomenclatural type ${ }^{\star}$ & Priority $\dagger$ & Reference \\
\hline Arthrobacter sanguinis Mages et al. 2009 & sp. nov. & Strain CCUG 46407 (=DSM 21259) & 1 & 7 \\
\hline Bacillus alkalinitrilicus Sorokin et al. 2009 & sp. nov. & Strain ANL-iso4 (=NCCB 100120=UNIQEM U240) & 2 & 10 \\
\hline Desulfovibrio aerotolerans Mogensen et al. 2009 & sp. nov. & Strain DvO5 (=DSM 16695=JCM 12613) & 3 & 9 \\
\hline Gordonia lacunae le Roes et al. 2009 & sp. nov. & Strain BS2 (=DSM 45085=NRRL B-24551) & 11 & 5 \\
\hline Leptospira licerasiae Matthias et al. 2009 & sp. nov. & Strain VAR $010(=$ ATCC BAA-1110=KIT VAR 010) & 6 & 8 \\
\hline Natronobacillus azotifigens Sorokin et al. 2009 & sp. nov. & Strain 24KS-1 (=NCCB 100215=UNIQEM U378) & 9 & 11 \\
\hline Nocardia blacklockiae Conville et al. 2009 & sp. nov. & Strain ATCC 700035 (=DSM 45135) & 5 & 2 \\
\hline Nocardia wallacei Conville et al. 2009 & sp. nov. & Strain ATCC 49873 (=DSM 45136) & 5 & 2 \\
\hline Nonomuraea candida le Roes and Meyers 2009 & sp. nov. & Strain HMC10 (=DSM 45086=NRRL B-24552) & 8 & 6 \\
\hline Schumannella An et al. 2009 & gen. nov. & Schumannella luteola An et al. 2009 & 10 & 1 \\
\hline Schumannella luteola An et al. 2009 & sp. nov. & Strain KHIA (=JCM 23215=TISTR 1824) & 10 & 1 \\
\hline Pelagibacillus goriensis Kim et al. 2007)§ & & & & \\
\hline
\end{tabular}

For references to Validation Lists 1-71, see Int J Syst Bacteriol 49 (1999) 1325. Lists 72-124 were published in Int J Syst Evol Microbiol 50 (2000) 3, 423, 949, 1415, 1699, 1953; and 51 (2001) 1, 263, 793, 1229, 1619, 1945; and 52 (2002) 3, 685, 1075, 1437, 1915; and 53 (2003) 1, 373, 627, 935, 1219, 1701; and 54 (2004) 1, 307, 631, 1005, 1425, 1909; and 55 (2005) 1, 547, 983, 1395, 1743, 2235; and 56 (2006) 1, 499, 925, 1459, 2025, 2507; and 57 (2007) 1, 433, 893, 1371, 1933, 2449; and 58 (2008) 1, 529, 1057, 1511, 1993, 2471.

*Abbreviations of culture collections cited in this list can be found at http://ijs.sgmjournals.org/misc/collections.dtl $\dagger$ Priority number assigned according to the date the documentation and request for validation are received.

$¥$ The culture collection accession number KIT VAR 010 has been provided on request for validation. The culture collection accession number WPR VAR 010 is also cited in the effective publication, but the authors did not provide a certificate of deposition from this collection.

$\$$ Terribacillus goriensis (Kim et al. 2007) Krishnamurthi and Chakrabarti 2008 appears in the October 2008 issue of the IJSEM. However, this name was not validly published [see Rule 27(3)]. Krishnamurthi and Chakrabarti propose to transfer Pelagibacillus goriensis Kim et al. 2007 (the type species of the genus Pelagibacillus) to the genus Terribacillus as Terribacillus goriensis comb. nov. According to Rule 37a, bacteriologists adhering to this proposal must change the name Pelagibacillus to Terribacillus. 


\section{References}

1. An, S.-Y., Xiao, T. \& Yokota, A. (2008). Schumannella luteola gen. nov., sp. nov., a novel genus of the family Microbacteriaceae. J Gen Appl Microbiol 54, 253-258.

2. Conville, P. S., Brown, J. M., Steigerwalt, A. G., Brown-Elliott, B. A. \& Witebsky, F. G. (2008). Nocardia wallacei sp. nov. and Nocardia blacklockiae sp. nov., human pathogens and members of the "Nocardia transvalensis complex". J Clin Microbiol 46, 1178-1184.

3. Johnson, D. B., Joulian, C., d'Hugues, P. \& Hallberg, K. B. (2008). Sulfobacillus benefaciens sp. nov., an acidophilic facultative anaerobic Firmicute isolated from mineral bioleaching operations. Extremophiles 12, 789-798.

4. Krishnamurthi, S. \& Chakrabarti, T. (2008). Proposal for transfer of Pelagibacillus goriensis Kim et al. 2007 to the genus Terribacillus as Terribacillus goriensis comb. nov. Int J Syst Evol Microbiol 58, 2287-2291.

5. le Roes, M., Goodwin, C. M. \& Meyers, P. R. (2008). Gordonia lacunae sp. nov., isolated from an estuary. Syst Appl Microbiol 31, 17-23.

6. le Roes, M. \& Meyers, P. R. (2008). Nonomuraea candida sp. nov., a new species from South African soil. Antonie Van Leeuwenhoek 93, 133-139.
7. Mages, I. S., Frodl, R., Bernard, K. A. \& Funke, G. (2008). Identities of Arthrobacter spp. and Arthrobacter-like bacteria encountered in human clinical specimens. J Clin Microbiol 46, 2980-2986.

8. Matthias, M. A., Ricaldi, J. N., Cespedes, M., Diaz, M. M., Galloway, R. L., Saito, M., Steigerwalt, A. G., Patra, K. P., Ore, C. V., Gotuzzo, E., Gilman, R. H., Levett, P. N. \& Vinetz, J. M. (2008). Human leptospirosis caused by a new, antigenically unique Leptospira associated with a Rattus species reservoir in the peruvian Amazon. PLoS Negl Trop Dis 2, e213. doi:10.1371/journal.pntd.0000213

9. Mogensen, G. L., Kjeldsen, K. U. \& Ingvorsen, K. (2005). Desulfovibrio aerotolerans sp. nov., an oxygen tolerant sulphatereducing bacterium isolated from activated sludge. Anaerobe 11, 339-349.

10. Sorokin, D. Y., van Pelt, S. \& Tourova, T. P. (2008). Utilization of aliphatic nitriles under haloalkaline conditions by Bacillus alkalinitrilicus sp. nov. isolated from soda solonchak soil. FEMS Microbiol Lett 288, 235-240.

11. Sorokin, I. D., Zadorina, E. V., Kravchenko, I. K., Boulygina, E. S., Tourova, T. P. \& Sorokin, D. Y. (2008). Natronobacillus azotifigens gen. nov., sp. nov., an anaerobic diazotrophic haloalkaliphile from soda-rich habitats. Extremophiles 12, 819-827. 